\title{
Stability and Convergence of the Peaceman-Rachford ADI Method for Initial-Boundary Value Problems
}

\author{
By W. H. Hundsdorfer and J. G. Verwer
}

\begin{abstract}
In this paper an analysis will be presented for the ADI (alternating direction implicit) method of Peaceman and Rachford applied to initial-boundary value problems for partial differential equations in two space dimensions. We shall use the method of lines approach. Motivated by developments in the field of stiff nonlinear ordinary differential equations, our analysis will focus on problems where the semidiscrete system, obtained after discretization in space, satisfies a one-sided Lipschitz condition with a constant independent of the grid spacing. For such problems, unconditional stability and convergence results will be derived.
\end{abstract}

1. Introduction. For many years splitting methods have proved valuable in the numerical solution of time-dependent, multi(space)-dimensional partial differential equations (PDE's). The general idea of splitting is to attack a multi-dimensional problem in such a way that only one-dimensional computations are required. This idea has led to the development of a great variety of so-called alternating direction implicit (ADI) methods, locally one-dimensional (LOD) or fractional step methods, and hopscotch type methods [6]. ADI methods were first introduced by Peaceman, Douglas and Rachford for the solution of parabolic (and elliptic) equations in two [13] and three [3] space variables. The present paper is devoted to a study of the stability and convergence properties of the original Peaceman-Rachford ADI method when applied to initial-boundary value problems.

The idea of splitting has to do with the time integration, rather than with the space discretization. This suggests to adopt the method of lines approach [9], which has the advantage that it enables us to formulate the Peaceman-Rachford (PR) method in a very compact way for a wide class of (two-dimensional) initial-boundary value problems, including nonlinear ones (method (2.2)). Another advantage is that it enables us to directly use ideas and results from the field of stiff ordinary differential equations (ODE's), which in the last years has witnessed interesting developments on nonlinear stability and convergence [1].

Let us give a brief outline of the paper. Section 2 is devoted to some preliminary results which are relevant for the remainder. There we link the PR method with an LOD type splitting method, based on the implicit midpoint rule. Loosely speaking, this ADI/LOD link (made before in [8]) reveals that, with respect to step-by-step stability, the ADI method will behave very much the same as the fully implicit

Received December 9, 1987.

1980 Mathematics Subject Classification (1985 Revision). Primary 65M10, 65M15, 65M20.

Key words and phrases. Numerical analysis, time-dependent PDE's, alternating direction implicit methods, Peaceman-Rachford method, method of lines, stability, error bounds. 
midpoint rule. By the notion of step-by-step stability we mean the stability of the ODE integration formula for evolving time (A-stability is such a property). This observation is of interest, since the implicit midpoint rule is known to possess unconditional stability properties for nonlinear, stiff ODE problems satisfying a one-sided Lipschitz condition. In the remainder of the paper, we therefore assume such a condition to hold for the semidiscrete system under consideration.

The stability analysis for the PR method is carried out in detail in Section 3. Our analysis concentrates on unconditional stability, by which we mean that no relation is assumed between the stepsize in time and the space grid refinement. We present a result valid for nonlinear, noncommuting splitting operators, which refutes to some extent the often expressed view that for step-by-step stability commutativity is crucial. In the same section we also point out, through a numerical illustration, that when implementing the PR method on the computer for nonlinear problems, care must be exercised in solving the arising systems of nonlinear algebraic equations. If this is not done with sufficient accuracy, then the stability may deteriorate severely. This observation is of practical significance, because in applications one often linearizes the problem, which always can be interpreted as carrying out, in a certain way, one step of the iterative Newton process. The point of view we take here is that in many cases instability is an artifact of the linearization, and not of the ADI scheme itself.

Sections 4 and 5 are devoted to full convergence properties of the PR scheme. Here we distinguish between nonlinear (Section 4) and linear (Section 5) problems. The prefix full means that we compare the numerical solution directly with the exact PDE solution. More specifically, the main objective of our convergence analysis is the order in time $p$ appearing in a global error bound of the type

$$
\left\|U_{n}-u_{h}\left(t_{n}\right)\right\| \leq C_{1} \tau^{p}+C_{2} \max _{t}\left\|\alpha_{h}(t)\right\|,
$$

where $U_{n}$ is the numerical solution at time $t=t_{n}, u_{h}\left(t_{n}\right)$ the PDE solution at $t=t_{n}$ restricted to the imposed space grid, $\alpha_{h}$ the spatial truncation error, and $C_{1}$ and $C_{2}$ are constants completely independent of the stepsize $\tau$ and the space grid refinement. This independency means that we examine unconditional convergence. In the nonlinear case we prove such convergence with order $p=1$, which is one less than the order on a fixed space grid. The discrepancy is caused by influence of the boundary conditions, not by lack of smoothness. Here the notions of local and global order reduction come into play, which are elucidated in an extensive discussion devoted to the linear case. There we present also convergence results with $p=2$ and briefly outline how the so-called Fairweather-Mitchell correction fits into the convergence theory.

2. Preliminaries. As mentioned already in the introduction, we follow in this paper the method of lines approach. This enables us to formulate the PeacemanRachford method in a compact way and, in addition, allows for the general treatment we aim at. In the present section we have collected some preliminary material. Subsection 2.1 deals with the time integration formula, while Subsection 2.2 contains information on semidiscrete problems. 
2.1. The Peaceman-Rachford Integration Formula. Consider the real Cauchy problem for the nonlinear ODE system

$$
\dot{U}=F(t, U), \quad 0 \leq t \leq T, \quad U(0)=U_{0},
$$

where $U_{0} \in \mathbf{R}^{M}$ and $F:[0, T] \times \mathbf{R}^{M} \rightarrow \mathbf{R}^{M}$ are given. This system is assumed to originate from spatial discretization of an initial-boundary value PDE problem. For the moment there is no need to be more specific about (2.1a). We suppose that $F$ can be decomposed into two simpler functions $F_{1}$ and $F_{2}$, with

$$
F_{1}(t, v)+F_{2}(t, v)=F(t, v) \text { for all }(t, v) \in[0, T] \times \mathbf{R}^{M} \text {. }
$$

The meaning of this linear splitting will become clear later.

The Peaceman-Rachford integration formula we examine in this paper is then given by

$$
\begin{aligned}
& U_{n+1 / 2}=U_{n}+\frac{1}{2} \tau F_{1}\left(t_{n+1 / 2}, U_{n+1 / 2}\right)+\frac{1}{2} \tau F_{2}\left(t_{n}, U_{n}\right), \\
& U_{n+1}=U_{n+1 / 2}+\frac{1}{2} \tau F_{1}\left(t_{n+1 / 2}, U_{n+1 / 2}\right)+\frac{1}{2} \tau F_{2}\left(t_{n+1}, U_{n+1}\right) .
\end{aligned}
$$

Here $t_{n+1 / 2}=t_{n}+\tau / 2, t_{n+1}=t_{n}+\tau$ for $n \geq 0$, and $U_{n+1 / 2}, U_{n+1}$ are the approximations to the exact solutions $U(t)$ of $(2.1)$ at time $t=t_{n+1 / 2}, t_{n+1}$, respectively. In this one-step integration formula $\left(U_{n} \rightarrow U_{n+1}\right), U_{n+1 / 2}$ is always considered to be an intermediate, auxiliary vector like in Runge-Kutta methods. In view of the one-step nature, it is easy to use variable stepsizes $\tau$. However, in the remainder we restrict ourselves to constant values of $\tau$.

From (2.2) two well-known integration methods can be recovered. If we put $F_{1}=0, F_{2}=F$, the trapezoidal rule is obtained, while for $F_{1}=F, F_{2}=0,(2.2)$ reduces to the implicit midpoint rule

$$
U_{n+1}=U_{n}+\tau F\left(t_{n+1 / 2}, \frac{1}{2} U_{n}+\frac{1}{2} U_{n+1}\right) .
$$

Inspection of (2.2) and (2.3) immediately reveals the characteristic features of (2.2). This method is alternately implicit in $F_{1}$ and $F_{2}$, whereas (2.3) is (fully) implicit in $F$. In our application of (2.2), following Peaceman and Rachford [13], $F$ stands for a discretized PDE operator in two space dimensions, and $F_{1}$ and $F_{2}$ are both assumed to be "one-dimensional". This implies that, per step, the costs involved in solving the implicit relations in (2.2) will be substantially lower than in a fully implicit method like (2.3).

Like the implicit midpoint and trapezoidal rule, the PR formula (2.2) has the (usual) order of consistency two for any given ODE system (2.1). This follows readily from a straightforward Taylor expansion. We mean here consistency with respect to the ODE solution $U$, not with respect to the underlying PDE solution. In Sections 4, 5 we will be more specific about consistency and convergence. There, we will compare the approximations $U_{n}$ directly with the PDE solution.

One of the points we wish to emphasize in this paper is that the stability of the PR method is in a sense governed by the stability of an implicit midpoint LOD method. To see this, we rewrite (2.2) in Euler fashion as

$$
\begin{aligned}
& Y_{n+1 / 2}=U_{n}+\frac{1}{2} \tau F_{2}\left(t_{n}, U_{n}\right), \\
& U_{n+1 / 2}=Y_{n+1 / 2}+\frac{1}{2} \tau F_{1}\left(t_{n+1 / 2}, U_{n+1 / 2}\right), \\
& Y_{n+1}=U_{n+1 / 2}+\frac{1}{2} \tau F_{1}\left(t_{n+1 / 2}, U_{n+1 / 2}\right), \\
& U_{n+1}=Y_{n+1}+\frac{1}{2} \tau F_{2}\left(t_{n+1}, U_{n+1}\right)
\end{aligned}
$$


for $n \geq 0$. This can be rearranged as a first step

$$
Y_{1 / 2}=U_{0}+\frac{1}{2} \tau F_{2}\left(t_{0}, U_{0}\right)
$$

followed by

$$
\begin{gathered}
\left\{\begin{array}{l}
U_{n+1 / 2}=Y_{n+1 / 2}+\frac{1}{2} \tau F_{1}\left(t_{n+1 / 2}, U_{n+1 / 2}\right), \\
Y_{n+1}=U_{n+1 / 2}+\frac{1}{2} \tau F_{1}\left(t_{n+1 / 2}, U_{n+1 / 2}\right),
\end{array}\right. \\
\left\{\begin{array}{l}
U_{n+1}=Y_{n+1}+\frac{1}{2} \tau F_{2}\left(t_{n+1}, U_{n+1}\right), \\
Y_{n+3 / 2}=U_{n+1}+\frac{1}{2} \tau F_{2}\left(t_{n+1}, U_{n+1}\right)
\end{array}\right.
\end{gathered}
$$

for $n \geq 0$. Note that now $(2.5 \mathrm{~b})$ constitutes the implicit midpoint rule

$$
Y_{n+1}=Y_{n+1 / 2}+\tau F_{1}\left(t_{n+1 / 2}, \frac{1}{2} Y_{n+1 / 2}+\frac{1}{2} Y_{n+1}\right) \text {. }
$$

Likewise, (2.5c) gives

$$
Y_{n+3 / 2}=Y_{n+1}+\tau F_{2}\left(t_{n+1}, \frac{1}{2} Y_{n+1}+\frac{1}{2} Y_{n+3 / 2}\right) .
$$

Consequently, apart from start and completion, the PR scheme is equivalent to an alternate application of the implicit midpoint schemes (2.6) and (2.7). The combination of these two is just a locally one-dimensional (LOD) method. It thus follows that this implicit midpoint LOD method governs the step-by-step stability of the PR method.

We note that the link between ADI and LOD has been made before by Gourlay and Mitchell [8]. We shall use it in our stability analysis presented in Section 3, although in a slightly different manner than above.

2.2. The Semidiscrete Problem. The stability and convergence analysis presented in the remainder of this paper is centered around the semidiscrete problem (2.1). This means that in a large part of our analysis there is no need to be specific about the underlying 2-dimensional PDE and its spatial discretization. The formulation (2.1) indicates that we have finite difference discretizations in mind, but finite element methods (continuous time Galerkin) could also be considered.

Let $\Omega_{h}$ be a space grid covering the spatial domain $\Omega \subset \mathbf{R}^{2}$ of the PDE. The vectors $U, F \in \mathbf{R}^{M}$ in (2.1) can be viewed as gridfunctions, each component (or set of components for nonscalar PDE's) corresponding to a value on a gridpoint of $\Omega_{h}$. The positive parameter $h$ refers to the grid distance, which may vary over the grid. In what follows, the limit $h \rightarrow 0$ means that the space grid is refined arbitrarily far in a suitable manner. We emphasize that the dimension $M$ of $U$ and $F$ depends on $h$. This dependence is suppressed in our notation. We assume that the boundary conditions on $\Gamma$, the boundary of $\Omega$, are incorporated in the function $F$.

Let $u(x, t)(x \in \Omega \cup \Gamma, t \in[0, T])$ be the exact PDE solution. The (pointwise) restriction of $u$ to $\Omega_{h}$ will be denoted by $u_{h}$. In our convergence analysis we will compare the fully discrete numerical solutions $U_{n}$ to $u_{h}\left(t_{n}\right)$. For this analysis we need the space truncation error $\alpha_{h}(t)$, defined by

$$
\alpha_{h}(t)=\dot{u}_{h}(t)-F\left(t, u_{h}(t)\right) \text { for } 0 \leq t \leq T .
$$

It will be assumed that $(2.1)$ is consistent with the underlying initial-boundary value problem, in the sense that

$$
\max _{0 \leq t \leq T}\left\|\alpha_{h}(t)\right\| \rightarrow 0 \quad \text { as } h \rightarrow 0 .
$$


Throughout this paper, $\|w\|$ denotes a chosen norm for $M$-dimensional vectors $w$, generated by an inner product $\langle v, w\rangle$ on $\mathbb{R}^{M}$. Likewise, we denote the induced matrix norm

$$
\|A\|=\sup _{w \neq 0}\|A w\| /\|w\|
$$

for $A \in L\left(\mathbb{R}^{M}\right)$, the space of real $M \times M$ matrices.

Our stability and convergence analysis will be restricted to semidiscrete problems satisfying the one-sided Lipschitz condition

$$
\left\langle F_{i}(t, \tilde{w})-F_{i}(t, w), \tilde{w}-w\right\rangle \leq \nu\|\tilde{w}-w\|^{2} \quad(i=1,2)
$$

for arbitrary $w, \tilde{w}$ in $\mathbb{R}^{M}$ and $0 \leq t \leq T$. Essential hereby is that the one-sided Lipschitz constant $\nu$ is independent of $h$, that is, of the grid spacing. We shall assume, for convenience, that $\nu \leq 0$, but this is not essential for what follows.

Condition (2.10) implies the exponential stability result

$$
\|\tilde{U}(t)-U(t)\| \leq e^{2 \nu t}\|\tilde{U}(0)-U(0)\|, \quad 0 \leq t \leq T,
$$

valid uniformly in $h$, for any pair of semidiscrete solutions $\tilde{U}, U$ of (2.1) [1]. It also implies that the spatial error $u_{h}(t)-U(t)$ satisfies the bound (cf. [19])

$$
\left\|u_{h}(t)-U(t)\right\| \leq \frac{e^{2 \nu t}-1}{2 \nu} \max _{0 \leq s \leq t}\|\alpha(s)\|, \quad 0 \leq t \leq T,
$$

provided $U(0)=u_{h}(0)$. Here one should read $t$ for $\left(e^{2 \nu t}-1\right) / 2 \nu$ in case $\nu=0$.

The well-posedness inequality (2.11) indicates that condition (2.10) is a fairly natural one. When combined with a suitable space discretization, interesting classes of PDE problems can be shown to satisfy (2.10). As an example we mention the semilinear heat equation

$$
u_{t}=\left(a_{1}(x, y, t) u_{x}\right)_{x}+\left(a_{2}(x, y, t) u_{y}\right)_{y}+s(x, y, t, u),
$$

with $a_{i}$ strictly positive and $\partial s / \partial u \leq \nu$. It is this type of equation for which ADI methods were originally developed.

As a word of warning, we should also note that many semidiscrete problems exist for which it may be very cumbersome to verify (2.10) for a certain norm, of course assuming such a norm exists. For example, solution-dependent coefficients $a_{i}$ in (2.13) cause difficulties here. Finally, the restriction that we let $w, \tilde{w}$ lie in the whole of $\mathbf{R}^{M}$ is not essential and is made only for convenience of presentation. In actual, nonlinear applications, it suffices to verify $(2.10)$ with $\tilde{w}=u_{h}(t)$ and $w$ lying in a tube around $u_{h}(t), 0 \leq t \leq T$.

To conclude this preliminary section, we recall that in the method of lines literature, semidiscrete PDE's are often treated as stiff ODE's [1], [16]. In fact, many results in the nonlinear stability theory for stiff ODE's relate to problems satisfying a one-sided Lipschitz condition like (2.10). Important parts in our stability and convergence analysis presented in the remainder of this paper do originate from the field of stiff ODE's.

3. Stability. The entire Section 3 is devoted to stability. We will present a stability result for the PR method (2.2) which is valid for any ODE system (2.1) satisfying the one-sided Lipschitz condition (2.10) (so, $F_{1}$ and $F_{2}$ may be nonlinear 
and noncommuting). For simplicity of presentation it will be assumed that (2.10) holds with $\nu \leq 0$. The results can be easily extended to the case $\nu>0$.

We will frequently use the following norm inequality for rational functions of matrices, basically due to von Neumann (cf. [1, Theorem 2.3.1]).

LEMMA 3.1. Let $r(z)$ be a rational function, and $A$ an $M \times M$ matrix satisfying $\langle A w, w\rangle \leq \nu\|w\|^{2}$ for all $w \in \mathbf{R}^{M}$. Then we have, for all $\tau>0$,

$$
\|r(\tau A)\| \leq \sup \{|r(z)|: z \in \mathbb{C}, \operatorname{Re}(z) \leq \tau \nu\} .
$$

3.1. A General Stability Inequality. Along with (2.2) we consider the perturbed PR scheme

$$
\begin{aligned}
& \tilde{U}_{n+1 / 2}=\tilde{U}_{n}+\frac{1}{2} \tau F_{1}\left(t_{n+1 / 2}, \tilde{U}_{n+1 / 2}\right)+\frac{1}{2} \tau F_{2}\left(t_{n}, \tilde{U}_{n}\right)+\tau \delta_{n+1 / 2} \\
& \tilde{U}_{n+1}=\tilde{U}_{n+1 / 2}+\frac{1}{2} \tau F_{1}\left(t_{n+1 / 2}, \tilde{U}_{n+1 / 2}\right)+\frac{1}{2} \tau F_{2}\left(t_{n+1}, \tilde{U}_{n+1}\right)+\tau \delta_{n+1} .
\end{aligned}
$$

The perturbations $\delta_{j}$ may stand for round-off errors, errors due to nonexactly solving the implicit relations, or for discretization errors. Let

$$
\varepsilon_{j}=\tilde{U}_{j}-U_{j} \text { for } j=n, n+1 / 2 \text { and } n \geq 0 .
$$

By subtracting (2.2) from (3.1) and using the mean value theorem, we obtain the following recursion for the errors,

$$
\begin{aligned}
& \varepsilon_{n+1 / 2}=\varepsilon_{n}+\frac{1}{2} \tau A_{1, n+1 / 2} \varepsilon_{n+1 / 2}+\frac{1}{2} \tau A_{2, n} \varepsilon_{n}+\tau \delta_{n+1 / 2}, \\
& \varepsilon_{n+1}=\varepsilon_{n+1 / 2}+\frac{1}{2} \tau A_{1, n+1 / 2} \varepsilon_{n+1 / 2}+\frac{1}{2} \tau A_{2, n+1} \varepsilon_{n+1}+\tau \delta_{n+1},
\end{aligned}
$$

where

$$
A_{i j}=\int_{0}^{1} F_{i}^{\prime}\left(t_{j}, \theta \tilde{U}_{j}+(1-\theta) U_{j}\right) d \theta, \quad F_{i}^{\prime}(t, w)=\partial F_{i}(t, w) / \partial w
$$

for $i=1,2$ and $j=n, n+1 / 2$. We now eliminate $\varepsilon_{n+1 / 2}$ from (3.3) to obtain

$$
\varepsilon_{n+1}=R_{n} \varepsilon_{n}+\tau \rho_{n+1}
$$

where

$$
\begin{aligned}
& R_{n}=\left(I-\frac{1}{2} \tau A_{2, n+1}\right)^{-1} r\left(\tau A_{1, n+1 / 2}\right)\left(I+\frac{1}{2} \tau A_{2, n}\right), \\
& \rho_{n+1}=\left(I-\frac{1}{2} \tau A_{2, n+1}\right)^{-1}\left[r\left(\tau A_{1, n+1 / 2}\right) \delta_{n+1 / 2}+\delta_{n+1}\right]
\end{aligned}
$$

and $r(z)=(1-z / 2)^{-1}(1+z / 2)$ is the familiar stability function of the implicit midpoint rule.

We note that as a consequence of the one-sided Lipschitz condition (2.10) with $\nu \leq 0$, all operations above are justified for arbitrary $\tau>0$. The implicit relations are uniquely solvable, and the following matrix norm inequalities follow from Lemma 3.1,

$$
\begin{aligned}
& \left\|r\left(\tau A_{i j}\right)\right\| \leq 1 \text { for all } \tau>0, \\
& \left\|\left(I-\frac{1}{2} \tau A_{i j}\right)^{-1}\right\| \leq 1 \text { for all } \tau>0 .
\end{aligned}
$$

This lemma also shows that when $\nu>0$, the upper bound 1 in (3.8) is to be replaced by $(1-\tau \nu / 2)^{-1}(1+\tau \nu / 2)$ and the corresponding range for $\tau$ by $\tau \nu<2$. Similarly, for $\nu<0$ the upper bound 1 in (3.9) can be sharpened to $(1-\tau \nu / 2)^{-1}$ for all $\tau>0$, while for $\nu>0$ this same bound holds for $\tau \nu<2$. Essential for 
application to PDE's of these inequalities is their validity uniformly in the mesh width of the space grid.

Direct application of (3.8) to obtain a bound for $\left\|R_{n}\right\|$ is not possible in general, because of the fact that $A_{2, n}$ may vary with $n$, and that $A_{2, n}$ and $A_{1, n+1 / 2}$ need not commute. Should $A_{2}$ be independent of $n$ and commute with the matrices $A_{1, n+1 / 2}$, then $R_{n}=r\left(\tau A_{2}\right) r\left(\tau A_{1, n+1 / 2}\right)$, so that $\left\|R_{n}\right\| \leq 1$ for arbitrary $\tau>0$. In this case we immediately derive from (3.5)-(3.9) the global error bound

$$
\left\|\varepsilon_{n}\right\| \leq\left\|\varepsilon_{0}\right\|+2 D \text { for all } \tau>0, n \geq 1,
$$

where $D$ is an upper bound for all $\left\|\delta_{j}\right\|, j=n, n+1 / 2$ and $n \geq 0$. This error bound expresses stability of the PR scheme with respect to initial errors $\varepsilon_{0}$ and perturbations $\delta_{j}$.

We now consider the general case (where the matrices do not commute and $A_{2, n}$ varies with $n$ ), and introduce the following transformation of the errors $\varepsilon_{n}$ for $n \geq 0$,

$$
\hat{\varepsilon}_{n}=\left(I-\frac{1}{2} \tau A_{2, n}\right) \varepsilon_{n} .
$$

These transformed errors satisfy

$$
\hat{\varepsilon}_{n+1}=\hat{R}_{n} \hat{\varepsilon}_{n}+\tau \hat{\rho}_{n+1},
$$

with

$$
\begin{aligned}
& \hat{R}_{n}=r\left(\tau A_{1, n+1 / 2}\right) r\left(\tau A_{2, n}\right), \\
& \hat{\rho}_{n+1}=r\left(\tau A_{1, n+1 / 2}\right) \delta_{n+1 / 2}+\delta_{n+1} .
\end{aligned}
$$

The effect of this transformation is that the new amplification operator $\hat{R}_{n}$ is factored into two operators, both with norm $\leq 1$, like in LOD methods [18], which gives us the global bound

$$
\left\|\hat{\varepsilon}_{n}\right\| \leq\left\|\hat{\varepsilon}_{0}\right\|+\max _{1 \leq k \leq n}\left\|\hat{\rho}_{k}\right\| \text { for all } \tau>0, n \geq 1 .
$$

Since $\left\|\varepsilon_{n}\right\| \leq\left\|\hat{\varepsilon}_{n}\right\|$ and $\left\|\hat{\rho}_{n+1}\right\| \leq\left\|\delta_{n+1 / 2}\right\|+\left\|\delta_{n+1}\right\|$ (cf. (3.8), (3.9)), we obtain the following stability result.

THEOREM 3.2. Consider (2.2) and (3.1) with perturbations $\left\|\delta_{j}\right\| \leq D$. Suppose the one-sided Lipschitz condition (2.10) holds with $\nu \leq 0$. Then the errors $\varepsilon_{n}=$ $\tilde{U}_{n}-U_{n}$ satisfy

$$
\left\|\varepsilon_{n}\right\| \leq\left\|\left(I-\frac{1}{2} \tau A_{2,0}\right) \varepsilon_{0}\right\|+2 D \text { for all } \tau>0, n \geq 1,
$$

where $A_{2,0}$ is given by (3.4).

The transformation (3.11) leading to this result is inspired by the ADI-LOD link outlined in Subsection 2.2. For linear problems with constant coefficients a similar result was obtained by Douglas and Gunn [2].

The bound (3.16) expresses stability of the PR scheme with respect to the transformed initial error $\hat{\varepsilon}_{0}$ and the original perturbations $\delta_{j}$. We will comment on $\left\|\hat{\varepsilon}_{0}\right\|$ in the next subsection. For the moment we note that if $\left\|\hat{\varepsilon}_{0}\right\| \leq C\left\|\varepsilon_{0}\right\|$ with $C>0$ independent of $h$ (for instance if $\varepsilon_{0}=0$ or $\varepsilon_{0}$ is a smooth gridfunction, so that $\left.\left\|A_{2,0} \varepsilon_{0}\right\| \leq C^{\prime}\left\|\varepsilon_{0}\right\|\right)$, then (3.16) shows unconditional stability without the common assumptions that $F_{1}$ and $F_{2}$ are linear and commuting. Note that when $A_{2, n}$ 
is independent of $n$ and commutes with $A_{1, n+1 / 2}$, no additional smoothness of $\varepsilon_{0}$ is required (cf. (3.10)).

3.2. Stabilization of the First Step. The transformed initial error $\hat{\varepsilon}_{0}=$ $\left(I-\frac{1}{2} \tau A_{2,0}\right) \varepsilon_{0}$ can be interpreted as the difference of two explicit Euler steps with negative stepsize $-\tau$,

$$
\hat{\varepsilon}_{0}=\left[\tilde{U}_{0}-\frac{1}{2} \tau F_{2}\left(t_{0}, \tilde{U}_{0}\right)\right]-\left[U_{0}-\frac{1}{2} \tau F_{2}\left(t_{0}, U_{0}\right)\right]
$$

In general, we may have $\left\|\hat{\varepsilon}_{0}\right\| \gg\left\|\varepsilon_{0}\right\|$, because of the explicitness in (3.17). Of course, if $\varepsilon_{0}$ is negligible, for instance if round-off is the only error source, then $\hat{\varepsilon}_{0}$ will be small for reasonable values of $h$. However, if $\varepsilon_{0}$ is not very small, for example if $\tilde{U}_{0}$ is obtained from experimental data with significant errors, then $\hat{\varepsilon}_{0}$ may become quite large and grow with spatial refinement. In such a situation we can stabilize the PR scheme by computing the first approximation $U_{1}$ by the backward Euler-LOD method, and apply (2.2) only for $n \geq 1$.

We thus consider the scheme with first step

$$
U_{1 / 2}=U_{0}+\tau F_{1}\left(t_{1 / 2}, U_{1 / 2}\right), \quad U_{1}=U_{1 / 2}+\tau F_{2}\left(t_{1}, U_{1}\right)
$$

and for $n=1,2,3, \ldots$,

$$
\begin{aligned}
& U_{n+1 / 2}=U_{n}+\frac{1}{2} \tau F_{1}\left(t_{n+1 / 2}, U_{n+1 / 2}\right)+\frac{1}{2} \tau F_{2}\left(t_{n}, U_{n}\right), \\
& U_{n+1}=U_{n+1 / 2}+\frac{1}{2} \tau F_{1}\left(t_{n+1 / 2}, U_{n+1 / 2}\right)+\frac{1}{2} \tau F_{2}\left(t_{n+1}, U_{n+1}\right) .
\end{aligned}
$$

On a fixed space grid the LOD scheme has only order 1 in time, but since we only perform one LOD step, the order of the process (3.18) will still be 2 on fixed space grids. Assume as before that (2.10) holds with $\nu \leq 0$. Repeating the stability analysis of the previous section, we now obtain

$$
\left\|\varepsilon_{n}\right\| \leq\left\|\left(I-\frac{1}{2} \tau A_{2,1}\right) \varepsilon_{1}\right\|+2 D \text { for all } \tau>0, n \geq 2
$$

with $D$ an upper bound for the $\left\|\delta_{j}\right\|(j=3 / 2,2,5 / 2, \ldots)$. The error $\varepsilon_{1}$ is now given by

$$
\varepsilon_{1 / 2}=\left(I-\tau A_{1,1 / 2}\right)^{-1}\left[\varepsilon_{0}+\tau \delta_{1 / 2}\right], \quad \varepsilon_{1}=\left(I-\tau A_{2,1}\right)^{-1}\left[\varepsilon_{1 / 2}+\tau \delta_{1}\right],
$$

where $\tau \delta_{1 / 2}, \tau \delta_{1}$ are perturbations on the right-hand side of (3.18a). By using Lemma 3.1, it follows that

$$
\begin{aligned}
\left\|\left(I-\frac{1}{2} \tau A_{2,1}\right) \varepsilon_{1}\right\| & \leq\left\|\left(I-\frac{1}{2} \tau A_{2,1}\right)\left(I-\tau A_{2,1}\right)^{-1}\right\|\left\|\varepsilon_{1 / 2}+\tau \delta_{1}\right\| \\
& \leq\left\|\varepsilon_{1 / 2}\right\|+\tau\left\|\delta_{1}\right\| \leq\left\|\varepsilon_{0}\right\|+\tau\left(\left\|\delta_{1 / 2}\right\|+\left\|\delta_{1}\right\|\right) .
\end{aligned}
$$

Thus we obtain for scheme (3.18) the stability result

$$
\left\|\varepsilon_{n}\right\| \leq\left\|\varepsilon_{0}\right\|+\tau\left(\left\|\delta_{1 / 2}\right\|+\left\|\delta_{1}\right\|\right)+2 D \text { for all } \tau>0, n \geq 1
$$

Amplification of $\varepsilon_{0}$ through $\hat{\varepsilon}_{0}$ is thus prevented.

We have no practical experience with scheme (3.18). In some numerical experiments with disturbed initial values, no large errors were found in the original PR scheme, so that there was little need for stabilization. We think that starting with one LOD step (or a few) may be advantageous in situations where $\varepsilon_{0}$ contains very high frequencies. Like the trapezoidal rule and implicit midpoint rule, the ADI scheme damps such high frequency error components very slowly, whereas the LOD scheme has strong damping properties [7], [20]. 
3.3. A Practical Observation. The PR scheme is implicit, and thus in actual application nonlinear algebraic equations have to be solved by an appropriate iterative method. Because the implicitness is "one-dimensional", it is feasible to implement a Newton-Raphson type method using a direct (band) solver for the arising linear systems, as it is customary in the field of stiff ODE's.

Let $\tilde{U}_{n}$ denote the numerical values generated by an implemented PR scheme in an actual application. These numerical values can be thought of as being solutions of the perturbed scheme (3.1), where the $\delta_{j}$ are errors arising from approximately solving the implicit relations, or, in other words, for stopping the iteration process. If the conditions of Theorem 3.1 hold, one can conclude that the implementation is stable if the stopping criterion is based on the residual test $\left\|\delta_{j}\right\| \leq$ [prescribed tolerance].

In applications one frequently circumvents the difficulties connected with the solution of nonlinear algebraic equations by applying linearization, which corresponds to using just one iteration, in one way or another, of a Newton-Raphson type iterative process. In the above setting this means that the $\delta_{j}$ are not controlled, and that, consequently, the stability may deteriorate. The following numerical example, quoted from van der Houwen and Sommeijer [10], serves to illustrate this phenomenon.

Consider the nonlinear parabolic equation

$$
u_{t}=3\left(u^{2} u_{x}\right)_{x}+3\left(u^{2} u_{y}\right)_{y}+x y u-9 t^{2}\left(x^{2}+y^{2}\right) u^{3}
$$

with exact solution $u(x, y, t)=\exp (x y t)$, on the unit square $0 \leq x, y \leq 1$ and $0 \leq t \leq 1$. We assume Dirichlet boundary conditions. On a uniform grid, with mesh width $h$ in both directions, we apply the difference formula (similar in $y$ direction)

$$
\left.\left(u^{2} u_{x}\right)_{x}\right|_{\left(x_{i}, y_{j}\right)} \approx h^{-2}\left\{u_{i+1 / 2, j}^{2} u_{i+1, j}-\left(u_{i+1 / 2, j}^{2}+u_{i-1 / 2, j}^{2}\right) u_{i j}+u_{i-1 / 2, j}^{2} u_{i-1, j}\right\}
$$

where $u_{i \pm 1 / 2, j}=\left(u_{i \pm 1, j}+u_{i, j}\right) / 2$. In the standard way, including equal distribution of the term $x y u-9 t^{2}\left(x^{2}+y^{2}\right) u^{3}$ over $F_{1}$ and $F_{2}$ and natural ordering of gridpoints, one can now set up the semidiscrete system (2.1) and apply scheme (2.2) for the time integration.

Table 3.1 shows the errors at $t=1$, measured in the discrete $L_{2}$-norm. In the left part, the errors are given for the case where only one iteration step of the Newton-Raphson process is used for solving the nonlinear algebraic equations, and the entries in the right part correspond to two iterations (which is sufficient for these $\tau, h$ values; more iterations do not alter accuracy). The deterioration of stability is clearly visible.

We emphasize once more that this deterioration of stability is an artifact of the chosen implementation. The experiment shows that the PR method itself is stable for the chosen values of $\tau$ and $h$. Unfortunately, we do not know whether the onesided Lipschitz condition (2.10) is valid, in some suitable norm, for this problem. Finally, it should be stressed that the present experiment does not stand on its own. One easily may conceive of more examples, see for instance [1, Section 9.4] for a related discussion. 
TABLE 3.1

The entries are $-{ }^{10} \log \|$ error at $t=1 \|$. The symbol $*$ denotes instability (overflow or near overflow).

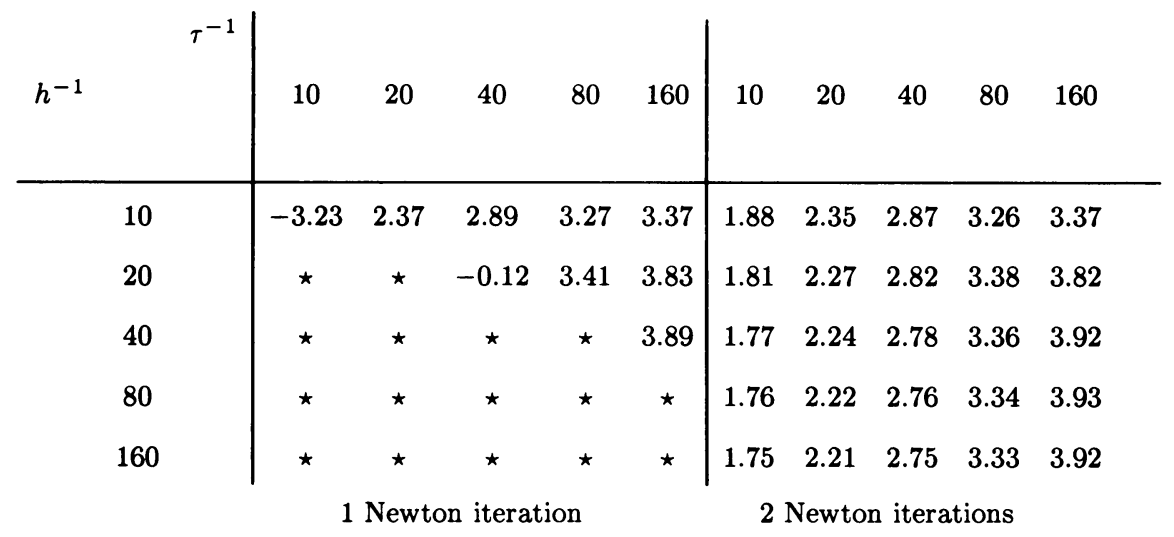

4. A General Convergence Result. The remainder of this paper is devoted to an investigation of the full convergence properties of the PR scheme (2.2). The prefix full indicates that we shall compare the fully discrete numerical solution $U_{n}$ directly to the exact PDE solution $u_{h}\left(t_{n}\right)$, without using the intermediate ODE solution $U\left(t_{n}\right)$. The main objective of our investigation is the order $p$ in time appearing in the general error bound

$$
\left\|u_{h}\left(t_{n}\right)-U_{n}\right\| \leq C_{1} \tau^{p}+C_{2} \max _{0 \leq t \leq T}\left\|\alpha_{h}(t)\right\| \quad\left(\text { for all } \tau, h>0,0 \leq t_{n} \leq T\right),
$$

where $C_{1}, C_{2}$ are constants independent of $\tau$ and $h$, and $\alpha_{h}$ is the spatial truncation error (2.8). Note that $\tau$ and $h$ are allowed to tend to zero simultaneously and independent of each other (unconditional convergence). We assume in the following that $U_{0}=u_{h}(0)$.

Convergence will be proved here by using the stability estimates of Section 3 for perturbations $\delta_{j}$. Let $\tilde{U}_{j}=u_{h}\left(t_{j}\right)$ for $j=n, n+1 / 2$ and $n \geq 0$. The $\delta_{j}$ then stand for residual discretization errors, and the $\varepsilon_{n}=u_{h}(t)-U_{n}$ are global discretization errors. By a Taylor expansion of $u_{h}(t)$ around $t=t_{n+1 / 2}$ we obtain from the first equation in (3.1)

$$
\begin{aligned}
\delta_{n+1 / 2}= & \frac{1}{2} \dot{u}_{h}\left(t_{n+1 / 2}\right)-\frac{1}{8} \tau \ddot{u}_{h}\left(s_{n+1 / 2}\right) \\
& -\frac{1}{2} F_{1}\left(t_{n+1 / 2}, u_{h}\left(t_{n+1 / 2}\right)\right)-\frac{1}{2} F_{2}\left(t_{n}, u_{h}\left(t_{n}\right)\right)
\end{aligned}
$$

for some intermediate point $s_{n+1 / 2} \in\left(t_{n}, t_{n+1 / 2}\right)$. Since $\dot{u}_{h}(t)=F_{1}\left(t, u_{h}(t)\right)+$ $F_{2}\left(t, u_{h}(t)\right)+\alpha_{h}(t)$, it follows that

$$
\begin{aligned}
\delta_{n+1 / 2}= & -\frac{1}{8} \tau \ddot{u}_{h}\left(s_{n+1 / 2}\right) \\
& +\frac{1}{2}\left[F_{2}\left(t_{n+1 / 2}, u_{h}\left(t_{n+1 / 2}\right)\right)-F_{2}\left(t_{n}, u_{h}\left(t_{n}\right)\right)\right]+\frac{1}{2} \alpha_{h}\left(t_{n+1 / 2}\right) .
\end{aligned}
$$

In a similar way we get

$$
\begin{aligned}
\delta_{n+1}= & \frac{1}{8} \tau \ddot{u}_{h}\left(s_{n+1}\right) \\
& -\frac{1}{2}\left[F_{2}\left(t_{n+1}, u_{h}\left(t_{n+1}\right)\right)-F_{2}\left(t_{n+1 / 2}, u_{h}\left(t_{n+1 / 2}\right)\right)\right]+\frac{1}{2} \alpha_{h}\left(t_{n+1 / 2}\right),
\end{aligned}
$$

where $s_{n+1} \in\left(t_{n+1 / 2}, t_{n+1}\right)$. 
Since $u_{h}$ is the restriction to $\Omega_{h}$ of the exact PDE solution, the terms $\ddot{u}_{h}(s)$ are bounded uniformly in $h$. Assuming

$$
\left\|F_{2}\left(t+\tau, u_{h}(t+\tau)\right)-F_{2}\left(t, u_{h}(t)\right)\right\| \leq C \tau \quad(\text { for } \tau, h>0,0 \leq t \leq T-\tau)
$$

with constant $C>0$, the stability estimate (3.16) directly leads to the following convergence result.

THEOREM 4.1. Let $F_{1}, F_{2}$ satisfy the one-sided Lipschitz condition (2.10) with $\nu \leq 0$. Assume $u_{h} \in C^{2}[0, T]$ and (4.4) holds. Then there are $C_{1}, C_{2}>0$ such that

$$
\left\|u_{h}\left(t_{n}\right)-U_{n}\right\| \leq C_{1} \tau+C_{2} \max _{0 \leq t \leq T}\left\|\alpha_{h}(t)\right\| \quad\left(\text { for } \tau, h>0,0 \leq t_{n} \leq T\right)
$$

The assumption (4.4) is a natural one; since $F\left(t, u_{h}(t)\right)=\dot{u}_{h}(t)-\alpha_{h}(t)$, a bound as in (4.4) will hold in general for the whole gridfunction $F\left(t, u_{h}(t)\right)$, provided only that $u$ is smooth. So, what we assume in fact here, is that this smoothness property is maintained in the splitting of $F$.

The bound (4.5) only shows order $p=1$ in time, whereas the order on fixed space grids is known to be 2 . This discrepancy is caused by the fact that we have avoided bounds on partial derivatives of $F_{1}$ and $F_{2}$. These contain negative powers of the mesh width in space, so that an error bound based on these quantities becomes useless when $h \rightarrow 0$. The material presented in the next section, where we examine linear problems, elucidates this point. There, we shall also derive bounds (4.1) with $p=2$.

5. Convergence for Linear Problems with Constant Coefficients. In the following we restrict our attention to initial-boundary value problems where the differential operators in space are linear and constant in time. The semidiscrete system then becomes

$$
\dot{U}=f(t, U)=A U+g(t)
$$

where $A$ is constant. We assume that $A$ can be split, in a natural way, into $A_{1}+A_{2}$, and (cf. (2.10))

$$
\left\langle A_{i} v, v\right\rangle \leq 0 \text { for all } v \in \mathbb{R}^{M} \text { and } i=1,2 .
$$

The inhomogeneous term $g(t)$ will contain two contributions,

$$
g(t)=b(t)+f(t)
$$

Here, $b(t)=b_{1}(t)+b_{2}(t)$ is assumed to emanate from the boundary conditions, and $f(t)$ represents a source term. For $f$ we shall consider splittings $f_{1}(t)=\theta f(t)$, $f_{2}(t)=(1-\theta) f(t)$ with $\theta \in[0,1]$, and $F_{i}(t, v)=A_{i} v+b_{i}(t)+f_{i}(t)$ for $v \in \mathbf{R}^{M}$, $t \in[0, T]$. Note that $A_{1}, A_{2}$ need not commute.

In the remaining subsections $O\left(\tau^{p} h^{k}\right)$ will be used to denote a scalar, or vector, whose absolute value, or norm, is bounded by $C \tau^{p} h^{k}$ for all possible $\tau$ and $h$, with $C>0$ a constant independent of $\tau, h$. This notation will also be used for $k=0$; $O\left(\tau^{p}\right)$ thus stands for a term which can be bounded by $C \tau^{p}$ uniformly for $h>0$.

5.1. The Structure of the Local Discretization Error. In this subsection we shall derive, by using the residual errors $\delta_{j}$, an expression for the discretization error 
which is introduced in the PR-process (2.2) in one single step. By expanding the formula (4.2) for $\delta_{n+1 / 2}$ somewhat further, we get

$$
\begin{aligned}
\delta_{n+1 / 2}= & -\frac{1}{8} \tau \ddot{u}_{h}\left(t_{n+1 / 2}\right)+\frac{1}{4} \tau \dot{F}_{2}\left(t_{n+1 / 2}, u_{h}\left(t_{n+1 / 2}\right)\right)+\frac{1}{2} \alpha_{h}\left(t_{n+1 / 2}\right) \\
& +\frac{1}{48} \tau^{2} \dddot{u}_{h}\left(s_{n+1 / 2}^{\prime}\right)-\frac{1}{16} \tau^{2} \ddot{F}_{2}\left(s_{n+1 / 2}^{\prime \prime}, u_{h}\left(s_{n+1 / 2}^{\prime \prime}\right)\right)
\end{aligned}
$$

for certain intermediate points $s_{n+1 / 2}^{\prime}, s_{n+1 / 2}^{\prime \prime} \in\left(t_{n}, t_{n+1 / 2}\right)$. Note that now total derivatives with respect to $t$ of $F_{2}\left(t, u_{h}(t)\right)$ come into play. Instead of (4.4) we shall impose in the following the slightly stronger condition $\ddot{F}_{2}\left(t, u_{h}(t)\right)=O(1)$, which also holds for reasonable splittings, provided $u$ is smooth. Define for $t \in[0, T]$

$$
w_{h}(t)=-\frac{1}{8} \ddot{u}_{h}(t)+\frac{1}{4} \dot{F}_{2}\left(t, u_{h}(t)\right) .
$$

We then obtain, assuming $u_{h}(t)$ to be three times continuously differentiable,

$$
\delta_{n+1 / 2}=\tau w_{h}\left(t_{n+1 / 2}\right)+\frac{1}{2} \alpha_{h}\left(t_{n+1 / 2}\right)+O\left(\tau^{2}\right) .
$$

Similarly,

$$
\delta_{n+1}=-\tau w_{h}\left(t_{n+1 / 2}\right)+\frac{1}{2} \alpha_{h}\left(t_{n+1 / 2}\right)+O\left(\tau^{2}\right) .
$$

With our choice $\tilde{U}_{j}=u_{h}\left(t_{j}\right)$, the error $\tau \rho_{n+1}$ defined in (3.5) represents the discretization error introduced in one single step of the process (2.2) (local discretization error). Since $A_{1}$ and $A_{2}$ are constant, we have

$$
\begin{aligned}
\tau \rho_{n+1} & =\left(I-\frac{1}{2} \tau A_{2}\right)^{-1}\left[r\left(\tau A_{1}\right) \tau \delta_{n+1 / 2}+\tau \delta_{n+1}\right] \\
& =\left(I-\frac{1}{2} \tau A_{2}\right)^{-1}\left(I-\frac{1}{2} \tau A_{1}\right)^{-1}\left[\left(I+\frac{1}{2} \tau A_{1}\right) \tau \delta_{n+1 / 2}+\left(I-\frac{1}{2} \tau A_{1}\right) \tau \delta_{n+1}\right] .
\end{aligned}
$$

From (5.5) and (3.9) we get the following result.

LEMMA 5.1. Consider the semidiscrete system (5.1) with $A_{1}, A_{2}$ satisfying (5.2). Suppose $u_{h} \in C^{3}[0, T]$ and $\left\|\ddot{F}_{2}\left(t, u_{h}(t)\right)\right\|=O(1)(0 \leq t \leq T)$. Then we have for the local discretization error

$$
\begin{aligned}
\tau \rho_{n+1}= & \left(I-\frac{1}{2} \tau A_{2}\right)^{-1}\left(I-\frac{1}{2} \tau A_{1}\right)^{-1}\left[\tau^{3} A_{1} w_{h}\left(t_{n+1 / 2}\right)+\tau \alpha_{h}\left(t_{n+1 / 2}\right)\right] \\
& +O\left(\tau^{3}\right) .
\end{aligned}
$$

It should be noted that (5.6) does not yield a bound $\left\|\tau \rho_{n+1}\right\|=O\left(\tau^{3}\right)+$ $O(\tau)\left\|\alpha_{h}\left(t_{n+1 / 2}\right)\right\|$ in general, since $A_{1} w_{h}(t)$ need not be $O(1)$ for $h \rightarrow 0$, unless the gridfunction $w_{h}(t)$ satisfies certain homogeneous boundary conditions imposed by $A_{1}$ (these conditions are unnatural; see for example Subsection 5.2 and [15], [21]). The eventual unboundedness of $A_{1} w_{h}(t)$ thus originates from the boundaries, and will not show up if one considers pure Cauchy problems with $\Omega=\mathbf{R}^{2}$.

We do have, in view of Lemma 3.1, $\left\|\left(I-\frac{1}{2} \tau A_{2}\right)^{-1}\right\| \leq 1$ and $\left\|\left(I-\frac{1}{2} \tau A_{1}\right)^{-1} \tau A_{1}\right\| \leq$ 2, which implies $\left\|\tau \rho_{n+1}\right\|=O\left(\tau^{2}\right)+O(\tau)\left\|\alpha_{h}\left(t_{n+1 / 2}\right)\right\|$. As we shall see in the next subsection, such a bound is nearly optimal. At first sight, this only leads to a global result $\left\|\varepsilon_{n}\right\|=O(\tau)+O(1) \max \left\|\alpha_{h}(t)\right\|$, and this was already established for nonlinear problems. In Subsection 5.3 it will be shown, however, that cancellation of errors may occur, which then leads to a second-order result $\left\|\varepsilon_{n}\right\|=O\left(\tau^{2}\right)+$ $O(1) \max \left\|\alpha_{h}(t)\right\|$. 
5.2. Local Error Analysis for a Simple Heat Equation. In this subsection we consider the inhomogeneous model problem

$$
\begin{aligned}
& u_{t}=u_{x x}+u_{y y}+s(x, y, t) \quad \text { on } \Omega, \\
& u(x, y, t)=u_{\Gamma}(x, y, t) \quad \text { on } \Gamma=\partial \Omega, \\
& u(x, y, 0)=u_{0}(x, y) \quad \text { on } \Omega \cup \Gamma
\end{aligned}
$$

where $0 \leq t \leq T$ and $\Omega$ is the unit square $(0,1) \times(0,1)$. Using standard space discretization on a uniform mesh, we obtain a system (5.1) for which precise bounds on the local errors can be given. First we describe the matrices $A_{1}, A_{2}$ and vectors $b_{1}, b_{2}, f$ appearing in the semidiscrete system.

Let $\Omega_{h}=\left\{\left(x_{i}, y_{j}\right): x_{i}=i h, y_{j}=j h, 1 \leq i, j \leq m\right\}$ with $h=1 /(m+1)$. We identify gridfunctions on $\Omega_{h}$ and vectors in $\mathbf{R}^{M}\left(M=m^{2}\right)$ in a natural way, assuming row-wise ordering on $\Omega_{h}$. Thus $w: \Omega_{h} \rightarrow \mathbf{R}^{M}$ will also be written as $w=$ $\left(w_{1}^{T}, \ldots, w_{m}^{T}\right)^{T}$ with $w_{j}=\left(w_{1 j}, \ldots, w_{m j}\right)^{T} \in \mathbf{R}^{m}, w_{i j}=w\left(x_{i}, y_{j}\right)$, and $w\left(x_{i}, y_{j}\right)$ will be called a component of the gridfunction $w$. Further, we shall use, for matrices and vectors, the direct (Kronecker) product $\otimes$ (see [12, Sections 12.1, 12.2] for standard properties). If $v=\left(v_{i}\right), \tilde{v}=\left(\tilde{v}_{i}\right) \in \mathbf{R}^{m}$, then $v \otimes \tilde{v}=\left(v_{1} \tilde{v}^{T}, \ldots, v_{m} \tilde{v}^{T}\right)^{T} \in$ $\mathbf{R}^{M}$ corresponds to the gridfunction with values $v_{j} \tilde{v}_{i}$ at $\left(x_{i}, y_{j}\right)$.

The matrices $A_{1}$ and $A_{2}$ can be written as

$$
A_{1}=-I \otimes Q, \quad A_{2}=-Q \otimes I,
$$

where $I$ is the $m \times m$ identity matrix, and $Q=h^{-2} \operatorname{tridiag}(-1,2,-1) \in L\left(\mathbf{R}^{m}\right)$ is the usual finite difference operator approximating $-\partial^{2} / \partial x^{2}$ in one dimension with Dirichlet boundary conditions (the first and last row of $Q$ contain nonzero entries $h^{-2}(2,-1)$ and $h^{-2}(-1,2)$, respectively). The boundary values are incorporated in $b(t)=b_{1}(t)+b_{2}(t) \in \mathbf{R}^{M}, b_{1}(t)$ having nonzero components $h^{-2} u_{\Gamma}(x \pm h, y, t)$ on $(x, y) \in \Omega_{h}$ adjacent to the vertical boundaries, and $b_{2}(t)$ with nonzero components $h^{-2} u_{\Gamma}(x, y \pm h, t)$ near the horizontal boundaries. Further, $f(t)$ is the restriction of $s(x, y, t)$ to $\Omega_{h}$.

The matrices $A_{1}$ and $A_{2}$ are symmetric and negative definite. Thus they satisfy (5.2) with respect to the standard inner product $\langle w, \tilde{w}\rangle=h^{2} w^{T} \tilde{w}$. Besides the norm $\|w\|=\langle w, w\rangle^{1 / 2}$ on $\mathbf{R}^{M}$, we also will use $|v|=\left(h v^{T} v\right)^{1 / 2}$ for $v \in \mathbb{R}^{m}$.

For the local errors we have (cf. (5.6))

$$
\begin{aligned}
\tau \rho_{n+1}= & \tau^{3}\left(I-\frac{1}{2} \tau A_{2}\right)^{-1}\left(I-\frac{1}{2} \tau A_{1}\right)^{-1} A_{1} w_{h}\left(t_{n+1 / 2}\right) \\
& +O(\tau) \alpha_{h}\left(t_{n+1 / 2}\right)+O\left(\tau^{3}\right),
\end{aligned}
$$

where $w_{h}(t)$, defined in (5.4), is a smooth gridfunction.

LEMMA 5.2. For any $\gamma \in[0,1 / 4)$ there is a constant $C_{\gamma}>0$ such that

$$
\left\|\tau^{3}\left(I-\frac{1}{2} \tau A_{2}\right)^{-1}\left(I-\frac{1}{2} \tau A_{1}\right)^{-1} A_{1} w_{h}(t)\right\| \leq C_{\gamma} \tau^{2+\gamma} \quad(\text { for } \tau, h>0,0 \leq t \leq T) .
$$

Proof. Since $\left\|\left(I-\frac{1}{2} \tau A_{2}\right)^{-1}\right\| \leq 1$, it is sufficient to prove the above bound for $\tau^{3}\left(I-\frac{1}{2} \tau A_{1}\right)^{-1} A_{1} w$ with $w=w_{h}(t)$. Let $\tilde{A}_{1}=-A_{1}$. This matrix is positive definite, and we can write for arbitrary $\gamma \in[0,1 / 4)$

$$
\left\|\tau^{3}\left(I-\frac{1}{2} \tau A_{1}\right)^{-1} A_{1} w\right\|=\tau^{2+\gamma}\left\|\left(I+\frac{1}{2} \tau \tilde{A}_{1}\right)^{-1}\left(\tau \tilde{A}_{1}\right)^{1-\gamma} \tilde{A}_{1}^{\gamma} w\right\| .
$$


The matrix $\left(I+\frac{1}{2} \tau \tilde{A}_{1}\right)^{-1}\left(\tau \tilde{A}_{1}\right)^{1-\gamma}$ is symmetric with eigenvalues contained in

$$
\left\{\left(1+\frac{1}{2} \tau \lambda\right)^{-1}(\tau \lambda)^{1-\gamma}: \lambda>0\right\} \subset(0,2),
$$

and thus its norm is bounded by 2 . We further have $\tilde{A}_{1}^{\gamma}=I \otimes Q^{\gamma}$. Hence, $\tilde{A}_{1}^{\gamma} w=\left(\left(Q^{\gamma} w_{1}\right)^{T}, \ldots,\left(Q^{\gamma} w_{m}\right)^{T}\right)^{T}$ and

$$
\left\|\tilde{A}_{1}^{\gamma} w\right\|^{2}=h^{2}\left(\tilde{A}_{1}^{\gamma} w\right)^{T}\left(\tilde{A}_{1}^{\gamma} w\right)=h \sum_{j=1}^{m}\left|Q^{\gamma} w_{j}\right|^{2} .
$$

It will be shown in the appendix that $\left|Q^{\gamma} w_{j}\right|$ is bounded uniformly for $h>0$ (with a bound only depending on smoothness properties of $w_{j}$, which are determined by smoothness of $u$, cf. (5.4)). Therefore, we also have $\left\|\tilde{A}_{1}^{\gamma} w\right\|=O(1)$, which completes the proof.

With the above lemma we obtain $\left\|\tau \rho_{n+1}\right\|=O\left(\tau^{q}\right)+O(\tau)\left\|\alpha_{h}\left(t_{n+1 / 2}\right)\right\|$ with $q \approx 2.25$. Note that this is only slightly better than the bound with $q=2$ which we derived directly from (5.6) for arbitrary problems (5.1) satisfying (5.2). In order to demonstrate the sharpness of these bounds, we consider the model problem (5.7) with boundary conditions $u_{\Gamma}=0$, initial value $u_{0}=0$, and source term

$$
s(x, y, t)=\phi(x) \phi(y)-t(\phi(x)+\phi(y)), \quad \phi(z)=\frac{1}{2} z(z-1)(0 \leq z \leq 1) .
$$

The exact solution is $u(x, y, t)=t \phi(x) \phi(y)$. Since $\ddot{u}_{h}=0$ and $b=0$, we have

$$
A_{1} w_{h}(t)=\frac{1}{4} A_{1} \dot{F}_{2}\left(t, u_{h}(t)\right)=\frac{1}{4} A_{1}\left[A_{2} \dot{u}_{h}(t)+\dot{f}_{2}(t)\right]
$$

with $f_{2}(t)=(1-\theta) f(t)$. Viewed as gridfunctions, $\dot{u}_{h}(t)$ and $\dot{f}(t)$ have values $\phi(x) \phi(y),-\phi(x)-\phi(y)$, respectively, for $(x, y) \in \Omega_{h}$. As vectors in $\mathbf{R}^{M}$, they can be written as

$$
\dot{u}_{h}(t)=v \otimes v, \quad \dot{f}(t)=-e \otimes v-v \otimes e,
$$

where $e=(1, \ldots, 1)^{T}, v=\left(v_{1}, \ldots, v_{m}\right)^{T} \in \mathbf{R}^{m}$ with $v_{i}=\frac{1}{2} i h(i h-1)(1 \leq i \leq m)$. We have $Q v=-e$. By using standard properties of direct products it follows from (5.8) that

$$
A_{1} A_{2} \dot{u}_{h}(t)=e \otimes e, \quad A_{1} \dot{f}(t)=-e \otimes e+v \otimes Q e .
$$

Since no space errors are present here (the solution is quadratic), relation (5.9) gives

$$
\tau \rho_{n+1}=\frac{1}{4}(1-\theta) \tau^{3}\left(I-\frac{1}{2} \tau A_{2}\right)^{-1}\left(I-\frac{1}{2} \tau A_{1}\right)^{-1}[v \otimes Q e]+O\left(\tau^{3}\right) .
$$

It follows that

$$
\tau \rho_{n+1}=\frac{1}{4}(1-\theta) \tau^{3}\left[\left(I+\frac{1}{2} \tau Q\right)^{-1} v \otimes\left(I+\frac{1}{2} \tau Q\right)^{-1} Q e\right]+O\left(\tau^{3}\right)
$$

and finally

$$
\left\|\tau \rho_{n+1}\right\|=\frac{1}{4}(1-\theta) \tau^{3}\left|\left(I+\frac{1}{2} \tau Q\right)^{-1} v\right|\left|\left(I+\frac{1}{2} \tau Q\right)^{-1} Q e\right|+O\left(\tau^{3}\right) .
$$

From $\left(I+\frac{1}{2} \tau Q\right)^{-1} v=v+\frac{1}{2} \tau\left(I+\frac{1}{2} \tau Q\right)^{-1} e=v+O(\tau)$, we see that there is a constant $C^{\prime}>0$ such that $\left|\left(I+\frac{1}{2} \tau Q\right)^{-1} v\right|>C^{\prime}$ whenever $\tau>0$ is sufficiently small. In the appendix it will be shown that there exists a $C^{\prime \prime}>0$ such that $\left|\left(I+\frac{1}{2} \tau Q\right)^{-1} Q e\right| \geq C^{\prime \prime} \tau^{-3 / 4}$ for all $h>0$ sufficiently small and $\tau / h^{2}$ bounded away from 0 . For such $\tau$ and $h$, and $\theta \neq 1$, we thus have

$$
\left\|\tau \rho_{n+1}\right\| \geq C \tau^{2.25}
$$

with $C>0$ independent of $\tau$ and $h$. 
In case $\theta=1$ no order reduction occurs for this specific example with $u_{\Gamma}=0$; we get $\left\|\tau \rho_{n+1}\right\|=O\left(\tau^{3}\right)$ as on fixed space grids, since then $w_{h}(t)$ vanishes near the vertical boundaries, so that $A_{1} w_{h}(t)=O(1)$. For more complicated examples with time-dependent boundary conditions, the order will reduce also if $\theta=1$.

The observation that inhomogeneous terms cause local order reduction seems to originate with D'Yakonov [4]. Fairweather and Mitchell [5] introduced a correction term which restores the local order; they considered (5.7) with $s=0$ and timedependent boundaries, but as we saw above, such a correction is also necessary if $u_{\Gamma}=0, s \neq 0$. The correction consists of replacing in the PR scheme $b_{1}(t)$ by $\bar{b}_{1}(t)=b_{1}(t)-c(t), c(t)$ being the correction term still to be specified. This corresponds to a change in the boundary values for the intermediate solution $U_{n+1 / 2}$. With this correction we derive, in the same way as before, the expression for local errors

$$
\begin{aligned}
\tau \rho_{n+1}= & \left(I-\frac{1}{2} \tau A_{2}\right)^{-1}\left(I-\frac{1}{2} \tau A_{1}\right)^{-1} \tau^{3} v_{h}\left(t_{n+1 / 2}\right) \\
& +O(\tau) \alpha_{h}\left(t_{n+1 / 2}\right)+O\left(\tau^{3}\right)
\end{aligned}
$$

where now

$$
v_{h}(t)=A_{1} w_{h}(t)+\tau^{-2} c(t)
$$

The gridfunction $w_{h}(t)$ is smooth, and thus all components of $A_{1} w_{h}(t)$ are $O(1)$, except those corresponding to a gridpoint adjacent to the vertical boundaries. Consider the gridpoint $\left(x_{1}, y_{j}\right)$ near the left boundary. There we have

$$
\left[A_{1} w_{h}(t)\right]_{1 j}=h^{-2}\left(-2 w_{1 j}(t)+w_{2 j}(t)\right) .
$$

The correction $c(t)$ can now be used to compensate for the missing value $w_{0 j}(t)$. Thus we define

$$
\begin{aligned}
c_{1 j}(t)= & \tau^{2} h^{-2} w_{0 j}(t) \\
w_{0 j}(t)= & -\frac{1}{8} \ddot{u}_{0 j}(t)+\frac{1}{4} h^{-2}\left(\dot{u}_{0 j+1}(t)-2 \dot{u}_{0 j}(t)+\dot{u}_{0 j-1}(t)\right) \\
& +\frac{1}{4}(1-\theta) \dot{s}_{0 j}(t)
\end{aligned}
$$

where $u_{0 j}(t)=u\left(0, y_{j}, t\right), s_{0 j}(t)=s\left(0, y_{j}, t\right)$. In a similar way we define $c_{m j}(t)$ to compensate for the missing values near the right boundary, and we take $c_{i j}(t)=0$ for gridpoints with $1<i<m$. This causes $v_{h}(t)=O(1)$, so that $\tau \rho_{n+1}=$ $O\left(\tau^{3}\right)+O(\tau) \alpha_{h}\left(t_{n+1 / 2}\right)$.

The correction (5.13) slightly differs from the one in [5]. The reason is that we started from the particular form for the local error with $w_{h}$ given by (5.4). Since $\left\|\left(I-\frac{1}{2} \tau A_{1}\right)^{-1} \tau A_{1}\right\|=O(1)$, it follows that (5.11) also holds with $v_{h}(t)=$ $A_{1} \tilde{w}(t)+\tau^{-2} c(t)$,

$$
\begin{aligned}
\tilde{w}(t)= & -\frac{1}{2} \tau^{-2}\left[u_{h}\left(t-\frac{1}{2} \tau\right)-2 u_{h}(t)+u_{h}\left(t+\frac{1}{2} \tau\right)\right] \\
& +\frac{1}{4} \tau^{-1}\left[F_{2}\left(t+\frac{1}{2} \tau, u_{h}\left(t+\frac{1}{2} t\right)\right)-F_{2}\left(t-\frac{1}{2} \tau, u_{h}\left(t-\frac{1}{2} \tau\right)\right)\right] .
\end{aligned}
$$

This leads to (5.13) with $\ddot{u}_{0 j}(t)$ and $\dot{u}_{0 j}(t)$ replaced by standard differences, which is then the same as the original correction of Fairweather and Mitchell [5]. Generalization of this boundary value correction to a large class of initial-boundary value problems can be found in [17].

5.3. Cancellation of Local Errors. Let $q$ be the order in time of the local discretization errors. One then naively expects order $p=q-1$ for the global errors, 
as a result of addition of all the local errors. For the example of Subsection 5.2 this would give $p=1.25$ only, instead of second-order in time as on a fixed space grid. In this subsection it will be shown that under suitable assumptions we still have $p=2$, because of cancellation of local errors. In other words, the local errors suffer from a reduction in order, but in the transition from local to global, this reduction is annihilated. A similar behavior can be observed with the implicit midpoint rule, and to some extent, with other Runge-Kutta methods [15], [21]. A comprehensive analysis for the implicit midpoint rule can be found in [11]. The proof of Theorem 5.3 below was inspired by this analysis.

We consider again the general linear problem (5.1), but it will be assumed now that there exists a constant $C>0$ (independent of $\tau, h$ ), such that

$$
\begin{aligned}
& \left\|A^{-1} A_{1}\right\| \leq C, \\
& \left\|R^{n}\right\| \leq C \text { for all } n \geq 0 \text { with } t_{n} \in[0, T] .
\end{aligned}
$$

Here $R=\left(I-\frac{1}{2} \tau A_{2}\right)^{-1} r\left(\tau A_{1}\right)\left(I+\frac{1}{2} \tau A_{2}\right)$ is the matrix governing stability of the PR scheme (cf. formula (3.6)). Thus (5.15) is a Lax-Richtmeyer type condition for stability [14]. If $A_{1}, A_{2}$ are commuting, negative definite matrices, then both (5.14) and (5.15) hold with $C=1$. The results below are thus applicable to the heat equation with standard space discretization on a uniform mesh, which we considered in the previous subsection.

THEOREM 5.3. Suppose the conditions of Lemma 5.1 are satisfied, and (5.14), (5.15) hold. Then there are constants $C_{1}, C_{2}>0$ such that

$$
\left\|u_{h}\left(t_{n}\right)-U_{n}\right\| \leq C_{1} \tau^{2}+C_{2} \max _{0 \leq t \leq T}\left\|\alpha_{h}(t)\right\| \quad\left(\text { for all } \tau, h>0,0 \leq t_{n} \leq T\right) .
$$

Proof. Consider the recursion for the global errors $\varepsilon_{n}=u_{h}\left(t_{n}\right)-U_{n}$,

$$
\varepsilon_{n+1}=R \varepsilon_{n}+\tau \rho_{n+1} \quad(n \geq 0)
$$

(cf. (3.5)). The local errors, given by (5.6), can be written as

$$
\tau \rho_{n+1}=\tau^{3}\left(I-\frac{1}{2} \tau A_{2}\right)^{-1}\left(I-\frac{1}{2} \tau A_{1}\right)^{-1} A_{1} w_{h}\left(t_{n+1}\right)+O(\tau) \alpha_{h}\left(t_{n+1 / 2}\right)+O\left(\tau^{3}\right) .
$$

Note that $w_{h}$ is evaluated here at $t=t_{n+1}$. For the proof of the theorem we may omit the terms $O(\tau) \alpha_{h}\left(t_{n+1 / 2}\right)+O\left(\tau^{3}\right)$, since these will give only a contribution $O(1) \max \left\|\alpha_{h}(t)\right\|+O\left(\tau^{2}\right)$ to the global bound.

We define, for all $n \geq 0$,

$$
\bar{\rho}_{n}=\tau A^{-1} A_{1} w_{h}\left(t_{n}\right), \quad \bar{\varepsilon}_{n}=\varepsilon_{n}+\tau \bar{\rho}_{n} .
$$

Using the equality $R-I=\left(I-\frac{1}{2} \tau A_{2}\right)^{-1}\left(I-\frac{1}{2} \tau A_{1}\right)^{-1} \tau A$, we get

$$
\rho_{n+1}=(R-I) \bar{\rho}_{n+1} \text {. }
$$

Therefore, the $\bar{\varepsilon}_{n}$ satisfy the recursion

$$
\bar{\varepsilon}_{n+1}=R \bar{\varepsilon}_{n}+\tau R\left(\bar{\rho}_{n+1}-\bar{\rho}_{n}\right) \quad(n \geq 0) .
$$

The stability assumption (5.15) provides the global estimate

$$
\left\|\bar{\varepsilon}_{n}\right\| \leq C\left\|\bar{\varepsilon}_{0}\right\|+C t_{n} \max _{k}\left\|\bar{\rho}_{k+1}-\bar{\rho}_{k}\right\| .
$$


Since we have, in view of $(5.14),\left\|\varepsilon_{k}-\bar{\varepsilon}_{k}\right\|=\left\|\tau \bar{\rho}_{k}\right\|=O\left(\tau^{2}\right)$ and $\left\|\bar{\rho}_{k+1}-\bar{\rho}_{k}\right\|=O\left(\tau^{2}\right)$ for all $k \geq 0$, the second-order result for $\left\|\varepsilon_{n}\right\|$ now follows.

Lest we miss the obvious, the introduction of the new errors $\bar{\varepsilon}_{n}, \bar{\rho}_{n}$ in the above proof is redundant if $A_{1} w_{h}(t)=O(1)$ uniformly for $h \rightarrow 0$. We can then prove (5.16) (by using the stability result (3.15)) without the assumptions (5.14) and (5.15). However, the material presented in Subsection 5.2 shows that in general $\left\|A_{1} w_{h}(t)\right\| \rightarrow \infty$ for $h \rightarrow 0$, unless the PDE solution $u_{h}$ and the inhomogeneous term $f_{2}$ meet additional conditions near the boundary $\Gamma$. These conditions are unnatural, in the sense that they are only imposed by the PR scheme and they are unrelated to smoothness of the PDE solution.

Convergence results for general ADI methods applied to linear initial-boundary value problems were obtained by Douglas and Gunn [2]. They considered homogeneous boundary conditions, in which case it can be shown that the local error of the PR method has the same order as the local error of the Crank-Nicolson type scheme

$$
\left(I-\frac{1}{2} \tau A\right) U_{n+1}=\left(I+\frac{1}{2} \tau A\right) U_{n}+\tau \phi_{n}
$$

with inhomogeneous term

$$
\phi_{n}=f_{1}\left(t_{n+1 / 2}\right)+\frac{1}{2} f_{2}\left(t_{n}\right)+\frac{1}{2} f_{2}\left(t_{n+1}\right)-\frac{1}{4} \tau A_{1}\left(f_{2}\left(t_{n+1}\right)-f_{2}\left(t_{n}\right)\right) .
$$

Because of this special inhomogeneous term, the same additional conditions show up if one tries to prove second-order convergence for this scheme. Moreover, the approach of Douglas and Gunn is hard to generalize for inhomogeneous boundary conditions.

As an illustration of the local order reduction and cancellation of local errors, we consider the simple problem

$$
u_{t}=(1+y) u_{x x}+u_{y y}+s(x, y, t)
$$

on the unit rectangle with Dirichlet boundary conditions. The source term and initial-boundary values are chosen such that the exact solution is $u(x, y, t)=$ $\exp (x+y+t)$. In space, standard discretization was used on a uniform mesh with grid distance $h$ in both directions. The PR scheme (2.2) was applied with $\tau=h$ and equal distribution of the source term $\left(f_{1}=f_{2}=f / 2\right.$; the choice $f_{1}=f, f_{2}=0$ leads to similar results). The following table shows the number of correct digits $-{ }^{10} \log \left\|\varepsilon_{n}\right\|$ for $n=1$ (local error) and $n=N, \tau N=1$ (global error).

\section{TABLE 5.1}

Errors for PR scheme applied to (5.18) with $\tau=h$.

\begin{tabular}{c|cccc}
$\tau^{-1}$ & 5 & 10 & 20 & 40 \\
\hline $\begin{array}{l}\text { local } \\
\text { error }\end{array}$ & 2.03 & 2.58 & 3.18 & 3.80
\end{tabular}

\begin{tabular}{c|cccc}
$\tau^{-1}$ & 5 & 10 & 20 & 40 \\
\hline $\begin{array}{c}\text { global } \\
\text { error }\end{array}$ & 1.68 & 2.20 & 2.76 & 3.35
\end{tabular}

One nicely sees second-order, approximately, for both local and global errors (increase of $\simeq 0.6$ upon step halving). We note that since the continuous operators $(1+y) \partial^{2} / \partial x^{2}$ and $\partial^{2} / \partial y^{2}$ do not commute, the matrices $A_{1}$ and $A_{2}$ will not commute either. Therefore, we do not know whether the stability condition (5.15) 
holds. The numerical results, however, indicate that the conclusions of Theorem 5.3 are valid here.

With a Fairweather-Mitchell type correction we would obtain third order locally, but still second order for the global errors. Although this correction technique does not increase the global order, the numerical results of Sommeijer et al. [17] indicate that in many cases the numerical approximations will become more accurate (the error constant $C_{1}$ in (5.16) may be smaller for corrected schemes).

Acknowledgment. The authors are grateful to Joke Blom for carrying out the numerical experiments.

Appendix. In this appendix some technical results will be derived concerning the finite difference operator approximating $-\partial^{2} / \partial x^{2}$, which were used in Subsection 5.2. Similar results for the continuous operator were obtained by Brenner, Crouzeix and Thomée (RAIRO Anal. Numér., v. 16, 1982, pp. 5-26). The discrete case has some minor additional difficulties.

Let $m \in \mathbf{N}$ and $h=1 /(m+1)$. Consider the $m \times m$ matrix

$$
Q=h^{-2}\left[\begin{array}{rrrrrrr}
2 & -1 & & & & \\
-1 & 2 & -1 & & & \\
& \cdot & . & . & & \\
& & . & & . & . & \\
& & & . & . & . & \\
& & & -1 & 2 & -1 \\
& & & & & -1 & 2
\end{array}\right] \text {. }
$$

This matrix has the eigenvalue-eigenvector decomposition

$$
Q=V \Lambda V^{-1}
$$

with

$$
V=\left[v_{1}, v_{2}, \ldots, v_{m}\right], \quad v_{j}=\sqrt{2}(\sin (j h \pi), \sin (2 j h \pi), \ldots, \sin (m j h \pi))^{T} \in \mathbf{R}^{m}
$$

and

$$
\Lambda=\operatorname{diag}\left(\lambda_{1}, \lambda_{2}, \ldots, \lambda_{m}\right), \quad \lambda_{j}=4 h^{-2} \sin ^{2}(j h \pi / 2) .
$$

We have $h v_{i}^{T} v_{j}=\delta_{i j}$ (Kronecker delta). Therefore, $h V^{T} V=I$, and

$$
Q=h V \Lambda V^{T} \text {. }
$$

On $\mathbf{R}^{m}$ we consider the inner product $(v, w)=h v^{T} w$ and norm $|w|=(w, w)^{1 / 2}$. For any $w \in \mathbf{R}^{m}$ and $\psi: \mathbb{C} \rightarrow \mathbb{C}$, analytic on the positive real axis, we have

$$
\psi(Q) w=\sum_{j=1}^{m}\left(v_{j}, w\right) \psi\left(\lambda_{j}\right) v_{j}
$$

and, since $\left(v_{i}, v_{j}\right)=\delta_{i j}$, it follows that

$$
|\psi(Q) w|^{2}=\sum_{j=1}^{m}\left|\left(v_{j}, w\right) \psi\left(\lambda_{j}\right)\right|^{2} .
$$

For any two real functions $f, g$ we will use the notation $f(x) \sim g(x)(x \downarrow 0)$ if there are positive numbers $C_{0}, C_{1}$ and $H$ such that $C_{0} g(x) \leq f(x) \leq C_{1} g(x)$ for $0<x \leq H$. Further, $x_{j}=j h$ for $j=1,2, \ldots, m$. 
LEMMA 1. Suppose $f \in C(0,1], \gamma \geq 0$ and $f(x) \sim x^{-\gamma}(x \downarrow 0)$. Then, for $h \downarrow 0$,

$$
h \sum_{j=1}^{m} f(j h) \sim \begin{cases}1 & \text { if } \gamma<1 \\ \log (1 / h) & \text { if } \gamma=1 \\ h^{1-\gamma} & \text { if } \gamma>1\end{cases}
$$

Proof. Since we can split $f$ into a monotonically decreasing part $\left(\sim x^{-\gamma}\right.$ for $x \downarrow 0)$ and a bounded remainder, it is clear that we may assume without loss of generality that $f$ itself is monotonically decreasing. Then

$$
h \sum_{j=1}^{m} f\left(x_{j}\right) \geq \int_{h}^{1} f(x) d x .
$$

On the other hand,

$$
h \sum_{j=1}^{m} f\left(x_{j}\right)=h f(h)+h \sum_{j=2}^{m} f\left(x_{j}\right) \leq h f(h)+\int_{h}^{1} f(x) d x .
$$

We have $h f(h) \sim h^{1-\gamma}(h \downarrow 0)$. The integral $\int_{h}^{1} f(x) d x$ is $\sim-\log h(h \downarrow 0)$ if $\gamma=1$, and $\sim 1+h^{1-\gamma}(h \downarrow 0)$ if $\gamma \neq 1$.

In the remainder, $e_{j}$ will stand for the vector in $\mathbf{R}^{m}$ with $j$ th component equal to 1 and the other components 0 . The vector $(1,1, \ldots, 1)^{T} \in \mathbf{R}^{m}$ will be denoted by $e$.

LEMMA 2. Let $\gamma \geq 0$. We have

$$
\sup _{h>0}\left|Q^{\gamma} e\right|<\infty \quad \text { if and only if } \gamma<1 / 4 \text {. }
$$

Proof. Since $Q e=h^{-2}\left(e_{1}+e_{m}\right)$, it follows that

$$
Q^{\gamma} e=h^{-2} Q^{\gamma-1}\left(e_{1}+e_{m}\right)=h^{-2} \sum_{j=1}^{m}\left[h\left(v_{j 1}+v_{j m}\right] \lambda_{j}^{\gamma-1} v_{j}\right.
$$

with $v_{j i}=\sqrt{2} \sin (i j h \pi)$ the $i$ th component of $v_{j}$. If $j$ is even, we have $v_{j 1}+v_{j m}=0$, while $v_{j 1}+v_{j m}=2 v_{j 1}$ if $j$ is odd. In the limit $h \downarrow 0$, we thus obtain

$$
\begin{aligned}
\left|Q^{\gamma} e\right|^{2} & =h^{-2} \sum_{j=1}^{m}\left|\left(v_{j 1}+v_{j m}\right) \lambda_{j}^{\gamma-1}\right|^{2} \sim 2 h^{-2} \sum_{j=1}^{m}\left|v_{j 1} \lambda_{j}^{\gamma-1}\right|^{2} \\
& =4^{2 \gamma-1} h^{2-4 \gamma} \sum_{j=1}^{m}\left|\sin (j h \pi) \sin (j h \pi / 2)^{2 \gamma-2}\right|^{2} \\
& =4^{2 \gamma} h^{1-4 \gamma} h \sum_{j=1}^{m} \cos ^{2}(j h \pi / 2) \sin (j h \pi / 2)^{4 \gamma-2} .
\end{aligned}
$$

From Lemma 1 we see that $\left|Q^{\gamma} e\right|^{2} \sim h^{1-4 \gamma}\left(1+h^{4 \gamma-1}\right)(h \downarrow 0)$ for $\gamma \neq 1 / 4$, whereas $\left|Q^{\gamma} e\right|^{2} \sim-\log h(h \downarrow 0)$ for $\gamma=1 / 4$.

LEMMA 3. Let $\phi \in C^{3}[0,1]$ and $w=\left(w_{1}, w_{2}, \ldots, w_{m}\right)^{T}$ with $w_{j}=\phi\left(x_{j}\right)$ for $1 \leq j \leq m, m \in \mathbf{N}$. Suppose $\gamma<1 / 4$. Then $\sup _{h>0}\left|Q^{\gamma} w\right|<\infty$.

Proof. We have

$$
Q w=\left(a_{1}+h^{-2} b_{1}, a_{2}, a_{3}, \ldots, a_{m}+h^{-2} b_{m}\right)^{T}
$$


with $a_{j}=h^{-2}\left(-\phi\left(x_{j-1}\right)+2 \phi\left(x_{j}\right)-\phi\left(x_{j+1}\right)\right) \approx-\phi^{\prime \prime}\left(x_{j}\right)$ and $b_{1}=\phi(0), b_{m}=\phi(1)$. Let $a=\left(a_{1}, a_{2}, \ldots, a_{m}\right)^{T}$. Then

$$
Q^{\gamma} w=Q^{\gamma-1} a+h^{-2} Q^{\gamma-1}\left[b_{1} e_{1}+b_{m} e_{m}\right] .
$$

Since $Q$ is positive definite with eigenvalues larger than 1 , the vector $Q^{\gamma-1} a$ is bounded, uniformly for $h>0$, for any $\gamma<1$. In the proof of Lemma 2 we saw that $h^{-2} Q^{\gamma-1} e_{j}(j=1$ or $m$ ) is also bounded uniformly in $h$, provided that $\gamma<1 / 4$.

COROLlaRY 4. Let $w$ be as in Lemma 3. For any $\gamma \in[0,1 / 4)$ there exists a constant $C_{\gamma}>0$ such that

$$
\left|\left(I+\frac{1}{2} \tau Q\right)^{-1} Q w\right| \leq C_{\gamma} \tau^{\gamma-1} \quad(\text { for all } \tau, h>0) .
$$

Proof. The matrix $\left(I+\frac{1}{2} \tau Q\right)^{-1}(\tau Q)^{1-\gamma}$ is symmetric with eigenvalues in the interval $(0,2)$, so that the norm of this matrix is bounded by 2 . Since $\left|Q^{\gamma} w\right|$ is uniformly bounded for $h>0$, the assertion follows from the inequality

$$
\left|\left(I+\frac{1}{2} \tau Q\right)^{-1} Q w\right| \leq \tau^{\gamma-1}\left|\left(I+\frac{1}{2} \tau Q\right)^{-1}(\tau Q)^{1-\gamma}\right|\left|Q^{\gamma} w\right| .
$$

LEMMA 5. Let $\alpha>0$, and assume $\tau / h^{2} \geq \alpha$. Then there exists a constant $C>0$ such that for all $\tau>0$

$$
\left|\left(I+\frac{1}{2} \tau Q\right)^{-1} Q e\right| \geq C \tau^{-3 / 4} .
$$

Proof. It is sufficient to consider $h>0$ sufficiently small. Let

$$
\mu(\tau, h)=\left|\left(I+\frac{1}{2} \tau Q\right)^{-1} Q e\right|^{2} .
$$

Similarly as in the proof of Lemma 2 , we obtain for $h \downarrow 0$

$$
\mu(\tau, h)=h^{-2} \sum_{j=1}^{m}\left|\left(v_{j 1}+v_{j m}\right)\left(1+\frac{1}{2} \tau \lambda_{j}\right)^{-1}\right|^{2} \sim 2 h^{-2} \sum_{j=1}^{m}\left|v_{j 1}\left(1+\frac{1}{2} \tau \lambda_{j}\right)^{-1}\right|^{2} .
$$

It follows that for arbitrary $\beta>0$ and $h>0$ sufficiently small,

$$
\mu(\tau, h) \geq 4\left(1+\frac{1}{2} \beta\right)^{-2} h^{-2} \sum_{j \in J_{\beta}} \sin ^{2}(j h \pi),
$$

where $J_{\beta}=\left\{j: \frac{1}{2} \beta \leq \tau \lambda_{j} \leq \beta\right\}$. We take $\beta=2 \alpha$, so that $\beta h^{2} / 4 \tau \leq 1 / 2$. Since $\tau \lambda_{j}=4 \tau h^{-2} \sin ^{2}(j h \pi / 2)$, the index $j$ belongs to $J_{\beta}$ if and only if

$$
\beta h^{2} / 8 \tau \leq \sin ^{2}(j h \pi / 2) \leq \beta h^{2} / 4 \tau,
$$

that is,

$$
2(\pi h)^{-1} \arcsin \sqrt{\beta h^{2} / 8 \tau} \leq j \leq 2(\pi h)^{-1} \arcsin \sqrt{\beta h^{2} / 4 \tau} .
$$

For each $j \in J_{\beta}$ we thus have $\sin ^{2}(j h \pi) \geq \beta h^{2} / 8 \tau$. Inspection of the graph of the arcsin function shows that the number of terms in $J_{\beta}$ is $\sim h^{-1} \sqrt{h^{2} / \tau}=\tau^{-1 / 2}$ (for $h \downarrow 0)$. From the above lower bound for $\mu(\tau, h)$ it now follows that there exists a constant $C>0$ such that

$$
\mu(\tau, h) \geq C^{2} h^{-2}\left(h^{2} / \tau\right) \tau^{-1 / 2}=C^{2} \tau^{-3 / 2}
$$

for $h>0$ sufficiently small.

Remark. The upper and lower bounds of Corollary 4 and Lemma 5 also hold if $\left(I+\frac{1}{2} \tau Q\right)^{-1}$ is replaced by $\psi(\tau Q)$ with $\psi$ an arbitrary rational function satisfying $\psi(\infty)=0$ and $|\psi(z)| \leq 1$ for $z \in \mathbb{C}, \operatorname{Re} z \geq 0$. 
If $w$ is the restriction to $\left\{x_{j}\right\}$ of a smooth function $\phi:[0,1] \rightarrow \mathbb{R}$ with $\phi(0)=$ $\phi(1)=0$, then a bound as in Corollary 4 also holds for $\left|\phi(\tau Q) Q^{2} w\right|$ (sharpness then follows by considering $\phi(x)=x(x-1)$ ).

Centre for Mathematics and Computer Science

P. O. Box 4079

1009 AB Amsterdam, The Netherlands

1. K. DeKKer \& J. G. VerWer, Stability of Runge-Kutta Methods for Stiff Nonlinear Differential Equations, North-Holland, Amsterdam, 1984.

2. J. DoUglas, JR. \& J. E. GUNN, "A general formulation of alternating direction methods, Part I: Parabolic and hyperbolic problems," Numer. Math., v. 6, 1964, pp. 428-453.

3. J. DOUGLAS, JR. \& H. H. RACHFORD, JR., "On the numerical solution of heat conduction problems in two and three space variables," Trans. Amer. Math. Soc., v. 82, 1956, pp. 421-439.

4. E. G. D'YAKONOV, "Difference schemes with splitting operator for multi-dimensional nonstationary problems," Zh. Vychisl. Mat. i Mat. Fiz., v. 2, 1962, pp. 549-568.

5. G. FAIRWEATher \& A. R. Mitchell, "A new computational procedure for A.D.I. methods," SIAM J. Numer. Anal., v. 4, 1967, pp. 163-170.

6. A. R. GourlaY, "Splitting methods for time dependent partial differential equations," The State of the Art in Numerical Analysis (D. Jacobs, ed.), Academic Press, New York, 1977, pp. 757-791.

7. A. R. GoURLAY \& J. LL. MORRIS, "The extrapolation of first order methods for parabolic partial differential equations II," SIAM J. Numer. Anal., v. 17, 1980, pp. 641-655.

8. A. R. Gourlay \& A. R. MitChelL, "The equivalence of certain alternating direction and locally one-dimensional difference methods," SIAM J. Numer. Anal., v. 6, 1969, pp. 37-46.

9. P. J. VAN DER HOUWEN \& J. G. VERWER, "One-step splitting methods for semi-discrete parabolic equations," Computing, v. 22, 1979, pp. 291-309.

10. P. J. VAN DER HOUWEN \& B. P. SOMMEIJER, Improving the Stability of Predictor-Corrector Methods by Residue Smoothing, Report NM-R8707, Centre for Mathematics and Computer Science, Amsterdam, 1987.

11. H. F. B. M. KRAAIJEVANGER, " $B$-convergence of the implicit midpoint rule and the trapezoidal rule," BIT, v. 25,1985, pp. $652-666$.

12. P. Lancaster \& M. Tismenetsky, The Theory of Matrices, Academic Press, Orlando, 1985.

13. D. W. PEACEMAN \& H. H. RACHFORD, JR., "The numerical solution of parabolic and elliptic differential equations," J. Soc. Indust. Appl. Math., v. 3, 1955, pp. 28-41.

14. R. D. Richtmeyer \& K. W. MORTON, Difference Methods for Initial-Value Problems, Interscience, New York, 1967.

15. J. M. SAnZ-Serna, J. G. Verwer \& W. H. Hundsdorfer, "Convergence and order reduction of Runge-Kutta schemes applied to evolutionary partial differential equations," Numer. Math., v. 50, 1987, pp. 405-418.

16. J. M. SANZ-SERNA \& J. G. VERWER, "Stability and convergence in the stiff ODE/PDE interface," Appl. Numer. Math., v. 5, 1989, pp. 117-132.

17. B. P. SOMMEIJER, P. J. VAN DER HOUWEN \& J. G. VeRWER, "On the treatment of time-dependent boundary conditions in splitting methods for parabolic differential equations," Internat. J. Numer. Math. Engrg., v. 17, 1981, pp. 335-346.

18. J. G. VERWER, "Contractivity of locally one-dimensional splitting methods," Numer. Math., v. 44, 1984, pp. 247-259.

19. J. G. VERWER \& J. M. SANZ-SERNA, "Convergence of method of lines approximations to partial differential equations," Computing, v. 33, 1984, pp. 297-313.

20. J. G. VERWER \& H. B. DE VRIES, "Global extrapolation of a first order splitting method," SIAM J. Sci. Statist. Comput., v. 6, 1985, pp. 771-780.

21. J. G. VERWER, "Convergence and order reduction of diagonally implicit Runge-Kutta schemes in the method of lines," Numerical Analysis (D. F. Griffiths and G. A. Watson, eds.), Pitman Research Notes in Mathematics Series, vol. 140, 1986, pp. 220-237. 